\title{
Front Matter: Volume 6562
}

, "Front Matter: Volume 6562," Proc. SPIE 6562, Unattended Ground, Sea, and Air Sensor Technologies and Applications IX, 656201 (21 April 2009); doi: 10.1117/12.746441

SPIE Event: Defense and Security Symposium, 2007, Orlando, Florida, United SPIE. States 


\section{PROCEEDINGS OF SPIE}

\section{Unattended Ground, Sea, and Air Sensor Technologies and Applications IX}

\section{Edward M. Carapezza}

Editor

9-12 April 2007

Orlando, Florida, USA

Sponsored and Published by

SPIE-The International Society for Optical Engineering

Volume 6562

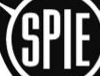

The International Society for Optical Engineering

Proceedings of SPIE-The International Society for Optical Engineering, 9780819466846, v. 6562

SPIE is an international technical society dedicated to advancing engineering and scientific applications of optical, photonic, imaging, electronic, and optoelectronic technologies. 
The papers included in this volume were part of the technical conference cited on the cover and title page. Papers were selected and subject to review by the editors and conference program committee. Some conference presentations may not be available for publication. The papers published in these proceedings reflect the work and thoughts of the authors and are published herein as submitted. The publisher is not responsible for the validity of the information or for any outcomes resulting from reliance thereon.

Please use the following format to cite material from this book:

Author(s), "Title of Paper," in Unattended Ground, Sea, and Air Sensor Technologies and Applications IX, edited by Edward M. Carapezza, Proceedings of SPIE Vol. 6562 (SPIE, Bellingham, WA, 2007) Article CID Number.

ISSN 0277-786X

ISBN 9780819466846

Published by

SPIE-The International Society for Optical Engineering

P.O. Box 10, Bellingham, Washington 98227-0010 USA

Telephone 1 360/676-3290 (Pacific Time) · Fax 1 360/647-1445

http://www.spie.org

Copyright (C) 2007, The Society of Photo-Optical Instrumentation Engineers

Copying of material in this book for internal or personal use, or for the internal or personal use of specific clients, beyond the fair use provisions granted by the U.S. Copyright Law is authorized by SPIE subject to payment of copying fees. The Transactional Reporting Service base fee for this volume is $\$ 18.00$ per article (or portion thereof), which should be paid directly to the Copyright Clearance Center (CCC), 222 Rosewood Drive, Danvers, MA 01923. Payment may also be made electronically through CCC Online at http://www.copyright.com. Other copying for republication, resale, advertising or promotion, or any form of systematic or multiple reproduction of any material in this book is prohibited except with permission in writing from the publisher. The CCC fee code is 0277 $786 \times / 07 / \$ 18.00$.

Printed in the United States of America. 


\section{Contents}

vii Conference Committee

\section{ACOUSTIC, SEISMIC, MAGNETICS AND MULTIMODAL SENSING}

656203 Passive ultrasonic method for human footstep detection [6562-02]

A. Ekimov, J. M. Sabatier, The Univ. of Mississippi (USA)

656204 Automated target recognition of humans in infrared images [6562-03]

D. Bankman, T. Neighoff, Johns Hopkins Univ. Applied Physics Lab. (USA)

656205 Personnel detection using ground sensors [6562-05]

R. Damarla, D. Ufford, Army Research Lab. (USA)

656207 Joint processing of vector-magnetic and acoustic-sensor data [6562-07]

R. J. Kozick, Bucknell Univ. (USA); B. M. Sadler, Army Research Lab. (USA)

656208 Acoustic sensing from small-size UAVs [6562-08]

D. N. Robertson, T. Pham, H. Edge, B. Porter, J. Shumaker, Army Research Lab. (USA);

D. Cline, Scientific Applications and Research Associates, Inc. (USA)

656209 Capabilities study of airborne acoustic sensor arrays [6562-10]

W. E. Prather, Miltec Research and Technology (USA); D. H. Bridges, Mississippi State Univ. (USA); T. Edwards, Raspet Flight Research Lab. (USA); D. S. Thompson, Mississippi State Univ. (USA)

\section{INVITED SESSION}

65620C An intelligent video framework for homeland protection (Invited Paper) [6562-57]

P. H. Tu, G. Doretto, N. O. Krahnstoever, A.G. A. Perera, F. W. Wheeler, X. Liu, J. Rittscher,

T. B. Sebastian, T. YU, K. G. Harding, GE Global Research (USA)

\footnotetext{
Pagination: Proceedings of SPIE follow an e-First publication model, with papers published first online and then in print and on CD-ROM. Papers are published as they are submitted and meet publication criteria. A unique, consistent, permanent citation identifier (CID) number is assigned to each article at the time of the first publication. Utilization of CIDs allows articles to be fully citable as soon they are published online, and connects the same identifier to all online, print, and electronic versions of the publication.

SPIE uses a six-digit CID article numbering system in which:

- The first four digits correspond to the SPIE volume number.

- The last two digits indicate publication order within the volume using a Base 36 numbering system employing both numerals and letters. These two-number sets start with 00, 01, 02, 03, 04, 05, 06, 07, 08, 09, OA, OB ... 0Z, followed by 10-1Z, 20-2Z, etc.

The CID number appears on each page of the manuscript. The complete citation is used on the first page, and an abbreviated version on subsequent pages.
} 
65620F Near earth propagation: physics revealed [6562-14]

R. Wert, A. Goroch, E. Worthington, V. Wong, Naval Research Lab. (USA)

65620G Energy saving in joint acoustic-video target detection and tracking [6562-15]

S. Zhang, G. A. Kahanda, CUNY College of Staten Island (USA)

656201 Metal Rubber materials and devices for flexible circuits, ground planes and interconnects [6562-17]

B. A. Davis, J. H. Lalli, A. Hill, R. O. Claus, NanoSonic, Inc. (USA)

\section{SIGNAL PROCESSING I}

65620J UGS video target detection and discrimination [6562-59]

G. M. Roberts, J. Fitzgerald, M. McCormack, R. Steadman, J. D. Vitale, Textron Systems

Corp. (USA)

65620K Capon beamspace beamforming for distributed acoustic arrays [6562-19]

N. J. Roseveare, Colorado State Univ. (USA); M. R. Azimi-Sadjadi, Information System

Technologies, Inc. (USA)

65620L Underwater source localization based on energy measurement with randomly distributed sensor array [6562-21]

X. Chen, U. Tureli, Stevens Institute of Technology (USA)

\section{SIGNAL PROCESSING II}

$65620 \mathrm{M}$ Loud target suppression: an innovative approach to reduce excessive sensor collaboration in distributed UGS systems [6562-23]

C. A. Stelzig, G. Lipelt, General Dynamics C4 Systems (USA); S. Minor, Army Night Vision and Electronic Sensors Directorate (USA)

$65620 \mathrm{~N}$ Role of quality of service metrics in visual target acquisition and tracking in resourceconstrained environments [6562-25]

M. Anderson, The Univ. of Alabama (USA); P. David, Army Research Lab. (USA)

656200 Distributed polarimetric sensing and processing (Invited Paper) [6562-26]

F. A. Sadjadi, Lockheed Martin Corp. (USA)

65620P A sparse undersea sensor network decision support system based on spatial and temporal random field [6562-27]

B. Ling, M. Zeifman, Migma Systems, Inc. (USA); M. Traweek, Office of Naval Research (USA); T. Wettergren, Naval Undersea Warfare Ctr. (USA)

65620Q Layered mode selection logic control for border security [6562-24]

T. Born, G. Ferrer, A. M. Wright, Hendrix College (USA); A. B. Wright, Univ. of Arkansas at Little Rock (USA) 
65620R Acoustic/seismic signal propagation and sensor performance modeling [6562-30] D. K. Wilson, Army Cold Regions Research and Engineering Lab. (USA); D. H. Marlin, Army Research Lab. (USA); S. Mackay, Atmospheric and Environmental Research, Inc. (USA)

65620 ARL participation in the C4ISR OTM experiment: integration and performance results [6562-31]

L. Zong, B. J. O'Brien, Army Research Lab. (USA)

65620T Modeling unmanned system collaborative target engagement [6562-32]

H. M. Jaenisch, dtech Systems Inc. (USA), and James Cook Univ. (Australia); J. W. Handley, Axiom Corp. (USA); M. L. Hicklen, dtech Systems Inc. (USA)

$65620 \mathrm{U}$ Geometric considerations for distribution of sensors in ad-hoc sensor networks [6562-33] T. Brown, D. Sarioz, A. Bar-Noy, CUNY Graduate School and Univ. Ctr. (USA); T. LaPorta, The Pennsylvania State Univ. (USA); D. Verma, IBM Thomas J. Watson Research Ctr. (USA); M. Johnson, CUNY Graduate School and Univ. Ctr. (USA); H. Rowaihy, The Pennsylvania State Univ. (USA)

\section{UNATTENDED GROUND SENSORS (UGS)}

65620Y Acoustic source localization with high performance sensor nodes [6562-37]

M. R. Azimi-Sadjadi, Information System Technologies, Inc. (USA); G. Kiss, B. Fehér, Budapest Univ. of Technology and Economics (Hungary); S. Srinivasan, Information System Technologies, Inc. (USA); Á. Lédeczi, Vanderbilt Univ. (USA)

$65620 Z$ iScout low-cost ad-hoc networked sensor enhancements [6562-38]

M. A. Winston, McQ, Inc. (USA)

656210 Detection of the under-soil intruder activity [6562-39]

J. Cechak, Univ. of Defence (Czech Republic)

656211 Low-cost unattended ground sensors for continuous surveillance [6562-58]

M. D. Perry, M. L. Pollard, D. S. Furuno, N. K. Askar, S. Benda, General Atomics (USA)

TRANSIENTS DETECTION

656212 Sniper detection using a helmet array: first tests in urban environment [6562-41]

S. Hengy, S. Demezzo, P. Hamery, ISL (France)

656213 Acoustic localization of mortar and small arms fires [6562-42]

T.-D. Tran-LuU, L. Solomon, S. Tenney, Army Research Lab. (USA)

656214 Quality of information trade-offs in the detection of transient phenomena [6562-43]

C. Bisdikian, IBM Thomas J. Watson Research Ctr. (USA)

656215 Acoustic classification of battlefield transient events using wavelet sub-band features [6562-44]

M. R. Azimi-Sadjadi, Information System Technologies, Inc. (USA); Y. Jiang, Univ. of

Colorado (USA); S. Srinivasan, Information System Technologies, Inc. (USA) 
656216 New seismic unattended small size module for footstep and light and heavy vehicles detection and identification [6562-04]

A. Pakhomov, T. Goldburt, General Sensing Systems, LLC (USA)

\section{KEYNOTE SESSION}

656217 DARPA NetCentric radio demonstration (Invited Paper) [6562-45]

L. B. Stotts, Defense Advanced Research Projects Agency (USA); S. Seidel, Raytheon (USA);

T. Krout, Communications Engineering for the Next Generation (USA); P. Kolodzy,

Consultant (USA)

\section{NETWORK SENSORS}

656218 Self-healing routing: a study in efficiency and resiliency of data delivery in wireless sensor networks [6562-46]

K. Wasilewski, J. W. Branch, M. Lisee, B. K. Szymanski, Rensselaer Polytechnic Institute (USA)

656219 Simulation of sensor networks using message queve infrastructure [6562-47]

D. C. Verma, IBM Thomas J. Watson Research Ctr. (USA); A. Stanford-Clark, IBM United Kingdom (United Kingdom)

65621A A survey of sensor selection schemes in wireless sensor networks [6562-48] H. Rowaihy, S. Eswaran, Pennsylvania State Univ. (USA); M. Johnson, CUNY (USA); D. Verma, IBM Thomas J. Watson Research Ctr. (USA); A. Bar-Noy, T. Brown, CUNY (USA); T. La Porta, Pennsylvania State Univ. (USA)

Author Index 


\title{
Conference Committee
}

\author{
Symposium Chair \\ John C. Carrano, Luminex Corporation (USA) \\ Symposium Cochair
}

Larry B. Stotts, Defense Advanced Research Projects Agency (USA)

Program Track Chair

Grant R. Gerhart, U.S. Army TARDEC/RDECOM (USA)

Conference Chair

Edward M. Carapezza, Defense Advanced Research Projects Agency (USA), DoD/DoJ Joint Program Committee Steering Group (USA), and University of Connecticut (USA)

Program Committee

John G. Blitch, ARACAR: Alliance for Robot Assisted Crisis Assessment and Response (USA)

John C. Carrano, Luminex Corporation (USA)

John S. Eicke, Army Research Laboratory (USA)

Todd M. Hintz, Space and Naval Warfare Systems Center, San Diego (USA)

Myron E. Hohil, U.S. Army Research, Development and Engineering Command (USA)

Ivan Kadar, Interlink Systems Sciences, Inc. (USA)

Michael A. Kolodny, Army Research Laboratory (USA)

Frank Patton, Defense Advanced Research Projects Agency (USA)

Tien Pham, Army Research Laboratory (USA)

Huub A. van Hoof, TNO (Netherlands)

Graeme van Voorthuijsen, TNO-FEL (Netherlands)

\section{Session Chairs}

1 Keynote Session

Edward M. Carapezza, Defense Advanced Research Projects Agency (USA), DoD/DoJ Joint Program Committee Steering Group (USA), and University of Connecticut (USA)

2 Acoustic, Seismic, Magnetics and Multimodal Sensing

Tien Pham, Army Research Laboratory (USA)

Graeme van Voorthuijsen, TNO-FEL (Netherlands) 
3 Joint Keynote Session with Conference 6538

Graeme van Voorthuijsen, TNO-FEL (Netherlands)

4a Invited Session

Edward M. Carapezza, Defense Advanced Research Projects Agency (USA), DoD/DoJ Joint Program Committee Steering Group (USA), and University of Connecticut (USA)

$4 \quad$ Enabling Technologies

Michael A. Kolodny, Army Research Laboratory (USA)

Tien Pham, Army Research Laboratory (USA)

$5 \quad$ Signal Processing I

Tien Pham, Army Research Laboratory (USA)

Graeme van Voorthuijsen, TNO-FEL (Netherlands)

$6 \quad$ Signal Processing II

Tien Pham, Army Research Laboratory (USA)

Graeme van Voorthuijsen, TNO-FEL (Netherlands)

$7 \quad$ Modeling and Simulation/Experimentation

Myron E. Hohil, U.S. Army Research, Development and Engineering Command (USA)

Tien Pham, Army Research Laboratory (USA)

$8 \quad$ Unattended Ground Sensors (UGS)

Michael A. Kolodny, Army Research Laboratory (USA)

9 Transients Detection

Myron E. Hohil, U.S. Army Research, Development and Engineering Command (USA)

Tien Pham, Army Research Laboratory (USA)

10 Keynote Session

Edward M. Carapezza, Defense Advanced Research Projects Agency (USA), DoD/DoJ Joint Program Committee Steering Group (USA), and University of Connecticut (USA)

11 Network Sensors

Tien Pham, Army Research Laboratory (USA)

Graeme van Voorthuijsen, TNO-FEL (Netherlands)

UGS Panel Discussion

Michael A. Kolodny, Army Research Laboratory (USA) 